\title{
Esquema de análisis de la Calidad de la información Contable ${ }^{1}$ Scheme of analysis of the Quality of the Accounting Information
}

DOI: http://dx.doi.org/10.17981/econcuc.40.1.2019.05

Artículo de investigación. Fecha de recepción: 30/12/2018 Fecha de aceptación: 13/03/2019

\author{
Julio Cesar Cantillo Padron \\ Universidad de Sucre. (Sincelejo, Colombia) \\ jccpsincel@gmail.com
}

Lucelly Rivero González

Agencia Nacional del Territorio - Universidad de Cartagena. (Sincelejo, Colombia)

lucelly.rivero2016@gmail.com

Para citar este artículo:

Cantillo, J. y Rivero L. (2019). Esquema de análisis de la Calidad de la información Contable. Económicas CUC, 40(1). 76-90. DOI: http:// doi.org/10.17981/econcuc.40.1.2019.05

\section{Resumen}

Dada las nuevas exigencias sobre información contable derivada por el crecimiento e internacionalización de empresas consideradas multinacionales, se aborda este artículo cuyo objetivo es presentar un esquema que permita analizar la incidencia de la adopción obligatoria de las Normas Internacionales de Información Financiera (NIIF) en la mejora de la calidad de la información contable en Colombia. Para lograrlo, primero se realizó una revisión bibliográfica, con el fin de discutir los conceptos y su evolución desde las ramas de la gestión organizacional, características y modelo de regulación normativo contable aplicable en Colombia y los atributos cualitativos de la información financiera, se presenta la propuesta, por último, se establece la forma de abordar el análisis de confiabilidad y de validez de la propuesta teniendo en cuenta la estadística descriptiva e inferencial, esto permitió establecer que resulta benéfico para las organizaciones utilizar la contabilidad financiera para la toma de decisiones, sin embargo al implementar las NIIF con medición de activos y pasivos bajo el método de valor razonable se establece que los resultados y la información pueden verse deformada. Se concluye que las investigaciones internacionales plantean una relación heterogénea entre la adopción de las normas internacionales y la calidad de la información contable, en razón a la complejidad y a los contextos legales, económicos, sociales y políticos de cada país.

Palabras clave: Contabilidad: gestión financiera: calidad; estados Financieros; información Financiera.

\begin{abstract}
Given the new demands on accounting information derived from the growth and internationalization of companies considered multinationals, this article is addressed whose objective is to present a schema that allows analyzing the incidence of the mandatory adoption of International Financial Reporting Standards (IFRS) in improving the quality of accounting information in Colombia. To achieve this, a bibliographic review was first carried out, in order to discuss the concepts and their evolution from the branches of organizational management, characteristics and accounting regulatory regulation applicable in Colombia and the qualitative attributes of the financial information, the proposal is presented, finally, it establishes the way to approach the analysis of reliability and validity of the proposal taking into account descriptive and inferential statistics, this allowed establishing that it is beneficial for organizations to use financial accounting to decision-making, however, when implementing IFRS with measurement of assets and liabilities under the fair value method, it is established that the results and information may be distorted. It is concluded that international investigations pose a heterogeneous relationship between the adoption of international standards and the quality of the information available, due to the complexity and legal, economic, social and political contexts of each country.
\end{abstract}

Keyworks: Accounting; financial management; quality; financial statements; financial information. 


\section{INTRODUCCIÓN}

La aparición y el desarrollo de las organizaciones multinacionales, el crecimiento de los mercados financieros, los avances tecnológicos y el comportamiento cambiante de los inversores contribuyo, entre otros factores, a la internacionalización de la actividad económica, esto ha generado que la información contable se extienda más allá de las fronteras de cada país y ha establecido mayores exigencias. La interpretación y la comprensión de la información contable a nivel internacional se ve obstaculizada por varios factores que inciden en la aplicación de los principios contables y de las normas que rigen la preparación de los informes.

Una década después de iniciado el proceso de implementación de las normas internacionales de información financiera se plantea que el impacto en la adopción de estos principios contables ha sido positivo (Callao, Ferrer, Jarne \& Laínez, 2010; Aguilar y Medina, 2013; Nobes \& Stanbler, 2015). Pero "estos argumentos no se pueden simplemente generalizar en el entorno actual" (Soderstrom \& Sun, 2007, pp. 36-37), autores como Morais, Fialho \& Dionísio (2018) han demostrado que la mejora en la calidad de la contabilidad no ha sido similar en todos los países" (p. 334).

En ese sentido, (Perafán \& Benavides, 2017) y (Barbel, 2008) plantean que los resultados han sido "heterogéneos". Las investigaciones contables internacional han señalado que "las mismas normas contables no determinan prácticas contables similares" (Nobes, 2006; Kvaal \& Nobes, 2010). Estas diferencias en la "práctica contable se derivan del hecho de que cada país tiene un conjunto di- verso de necesidades de información debido a los contextos legales, económicos, sociales y políticos únicos" (Ball, 2001; Brown, 2011).

Barth, Landsman \& Lang (2008) plantean que la adopción de las NIIF puede mejorar la calidad de la contabilidad, dado que reduce la discreción gerencial, permitiendo implementar de normas contables orientadas al mercado de capital y mejorando la relevancia del valor de los números contables. Sin embargo, también describen la calidad de la información contable puede verse afectada por un enfoque de gestión de ganancias. A nivel Latinoamericano se evidencian pocas investigaciones empíricas con respecto a Calidad de la información Contable, por lo tanto, se propone un esquema que permita analizar ¿Cómo La adopción obligatoria de las Normas Internacionales de Información Financiera mejora la calidad de la información contable en Colombia?

Teniendo en cuenta la transición reciente a las Normas Internacionales de Información Financiera y a partir de los requerimientos establecidos en Colombia, la propuesta busca contribuir a la discusión sobre los efectos de la adopción de las NIIF, sugiere el uso de un nuevo esquema que permita el análisis de posibles factores que inciden en la toma de decisiones. Es necesario resaltar que la propuesta no es meramente "reduccionistas y simplificadora" (Morin, 2001; Sanabria, Romero y Flórez, 2013), busca establecer dentro de la complejidad de las Ciencias Contables un punto de partida para nuevas propuestas o investigaciones que permitan la transformación o mejoramiento de las prácticas contables. 


\section{Fundamentos teóRICOS}

\section{Calidad y modelo de regulación}

La calidad es un aspecto importante en el Sistema de Información Contable (SIC), ya que puede posibilitar que los usuarios de la información tomen decisiones, sin embargo, se requiere que la información cumpla con tres aspectos importantes. La información debe ser oportuna, este aspecto se refiere a que la información debe estar disponible en los momentos que se requiera; suficiente, cuando se cumple con las necesidades de los usuarios y pertinente, las características de la información deben facilitar y permitir la toma de decisiones. Por tanto, una diferencia sustancial entre el "SIC (Sistema de Información Contable) y la realidad financiera puede desencadenar una serie de decisiones equivocadas que más adelante van a afectar a las organizaciones" (Cárdenas, Gallego y Tobón, 2018, p. 7).

El principio de calidad ha pasado por varios procesos evolutivos en el tiempo y este ha sido incorporado en los estados financieros, a través de los nuevos requerimientos. Es decir, se debe dar cumplimiento a una serie de características cualitativas y se debe tener presente el modelo de regulación contable y los usuarios de la información contable.

\section{- Evolución del concepto de Calidad}

El concepto de calidad ha evolucionado de la mano con la administración y la gestión organizacional, a partir de este proceso se ha modifica la forma de concebir la calidad, la cual ha estado asociado a la utilización de recursos. Según González (2009) la calidad ha evolucionado en etapas, la primera etapa denominada "Artesanal", cuyo objeto era "hacer las cosas bien independientemente del coste o esfuerzo necesario"; en la segunda etapa "Revolución industrial" (p. 5), lo importante fue satisfacer una gran demanda de bienes, el concepto de calidad estaba asociado a la producción en masa, la tercera desarrollada en medio de la "segunda guerra mundial" cuyo objetivo fue Asegurar la eficacia del armamento sin importar el costo, con la mayor y más rápida producción (Eficacia + Plazo = Calidad; la cuarta etapa "posguerra Japón" buscó mejorar la fabricación minimizando costos mediante la Calidad y Satisfaciendo al cliente (ser competitivo); seguidamente se incorpora el "Control de Calidad”, como Técnica de inspección en Producción para evitar la salida de bienes defectuosos y de esta manera se definen condiciones técnicas de los productos y por último, se establece la "Calidad Total" que busca satisfacer tanto al cliente externo como interno, es decir, ser altamente competitivo a través de la "Mejora Continua".

Teniendo en cuenta todas estos aspectos históricos no es posible establecer una definición universal de calidad, pero a partir de esta evolución se puede señalar que la calidad busca la "Satisfacción de las necesidades y expectativas" (Pérez, 1994) o según Alvarado, Hernandez y Chumaceiro (2010, p. 48) "el nivel o grado de respuesta que dan los empleados, funcionarios o servidores a las demandas y necesidades del cliente y/o usuario. Representa al mismo tiempo, la correcta utilización de los medios que permitan mantenerla”. Inicialmente fue concebida hacia la satisfacción del Cliente y posteriormente incorporada hacia los denominados Stakeholders, es decir 
se establece la necesidad de analizar los diferentes agentes que se benefician y afectan con la producción o prestación de un servicio (Entorno) y se establece como principio que todos los miembros de la organización son responsables de la calidad de los productos y de los servicios de la organización.

\section{- Órganos de Regulación Contable}

Varios órganos se encargan de emitir los estándares a nivel internacional, este es el caso del Consejo de Normas Internacionales de Contabilidad IASB (por sus siglas en ingles International Accounting Standard Board) el cual se encarga de emitir estándares contables aplicables al sector público, de igual forma, la Federación Internacional de Contadores - IFAC (por sus siglas en ingles International Federation Accountants), este último se encarga de emitir el código de ética profesional aplicable a los estándares, las Normas Internaciones de Aseguramiento y las Normas Internacionales de Contabilidad del Sector público.

No obstante, lo anterior en Colombia a través del Consejo Técnico de La Contaduría Pública, como órgano de normalización técnica de normas contables, de información financiera y de aseguramiento, se encarga de elaborar proyectos de normas que son sometidos a consideración a través de los Ministerios de Hacienda y Crédito Público y de Comercio, Industria y Turismo, estos ministerios son los encargados de emitir los respectivos decretos reglamentarios que definen los principios de reconocimiento, medición, revelación y presentación aplicables a sector privado.
- Modelo de regulación contable en Colombia

En los países latinoamericanos se "tienen una tradición enfáticamente legalista” (Gómez y Ospina, 2009, p. 14), es por eso que Colombia a través de la emisión de la (Ley 1314, 2009) modifica los principios de contabilidad generalmente aceptados y se adoptan las Normas Internacionales de Información Financiera, como "sistema único, homogéneo, de Alta Calidad y de forzosa observancia”. Este cambio implica un aparente avance en la presentación de la información financiera, cuyo objetivo es mejorar la calidad de la información financiera y la demanda de informes financieros con menor asimetrías, dado que esta información se ha enfocado mayoritariamente en la causación y liquidación de impuestos y/o contribuciones.

A partir de este requerimiento legal y de lo establecido en el (Decreto 2420, 2015) las organizaciones privadas en Colombia se categorizan en tres grandes grupos. El primer grupo, conformado por: emisores de valores, entidades de interés público y entidades con activos superiores a treinta mil (30.000) Salarios Mínimos Mensuales Legales Vigentes (en adelante SMMLV) o que cuente con un numero de personal superior a doscientos (trabajadores). Además, que cumplan con uno de los siguientes requisitos: ser subordinada o sucursal de compañía extranjera que aplique NIIF, ser subordinada o matriz de una compañía nacional que aplique NIIF, realizar importaciones o exportaciones que representen más del $50 \%$ de las compras del año inmediatamente anterior y ser matriz asociada o negocio conjunto de una o más entidades extranjeras que aplique NIIF. 
Por su parte, el (Decreto 2420, 2015) establece que el segundo grupo está conformado por las empresas que no cumplan con los requisitos del primer grupo y que cuenten con Activos totales por valor entre quinientos (500) y treinta mil (30.000) SMMLV o planta de personal entre once (11) y doscientos (200) trabajadores, además se permite pertenecer a este grupo las Microempresas con Activos totales (excluida la vivienda) por un valor máximo de quinientos (500) SMMLV o planta de personal no superior a los (10) trabajadores, y cuyos ingresos brutos anuales sean iguales o superiores a 6.000 SMMLV. Para el caso del tercer grupo, no debe cumplir con los requisitos del primer y segundo grupo; y además debe cumplir los criterios establecidos en el art. 499 del (Decreto 624, 1989) es necesario señalar que el tercer grupo es desarrollado solo en Colombia en comparación del resto de países latinoamericanos.
Caracteristicas y atributos cualitativos de la información financiera.

El concepto de calidad ha sido incorporado a los principios que regulan los estándares internacionales de información financiera, se deben destacar que el concepto de "calidad surge en medio del debate armonizador" (Zorio, García y Pucheta, 2004). Por tal motivo, la información contable debe cumplir con una serie de características necesarias para una adecuada toma de decisiones y debe "permitir formar juicios que faciliten la toma de decisiones" (Déniz, Bona, Pérez y Suárez, 2007, p. 135), es decir, "la información de los estados financieros debe reflejar la situación económica subyacente" (Chen, Tang, Jiang \& Lin, 2010).

Teniendo en cuenta que la contabilidad "está constituida por un conjunto de representaciones simbólicas, que impactan directamente la manera en que las

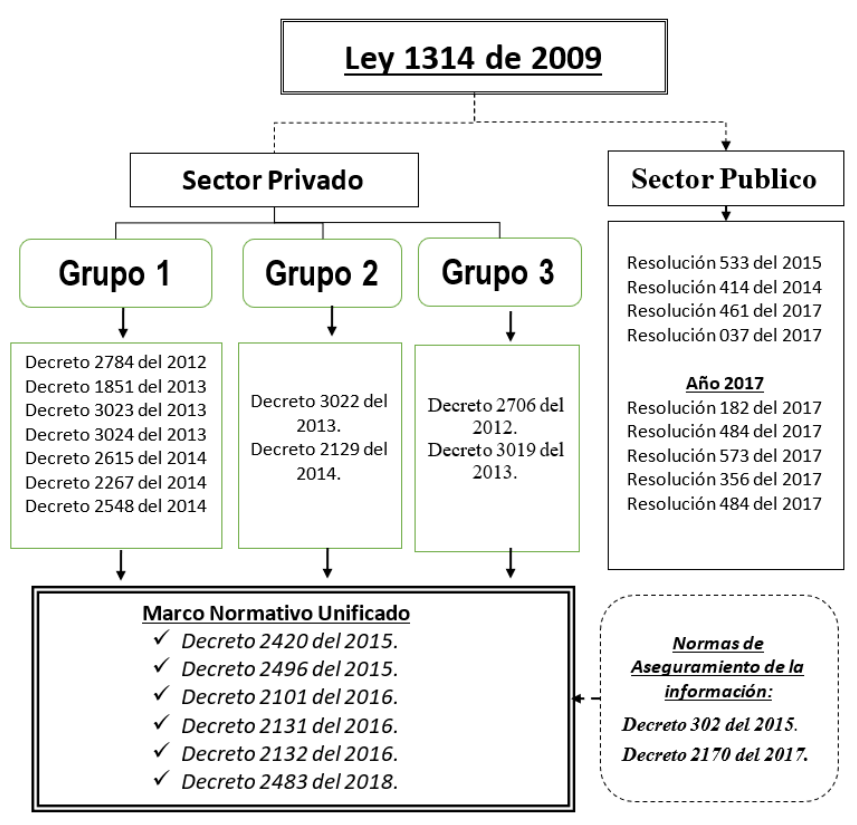

Figura 1. Marco normativo en Colombia.

Fuente: Elaboración propia a partir del marco normativo. 
organizaciones se relacionan con el entorno" (Villegas, 2003, p. 114) entidades como el Consejo de Normas Internacionales de Contabilidad (por sus siglas en ingles IASB) ha establecido una serie de atributos cualitativos que deben cumplir los estados financieros, para ser reconocidos de alta calidad. El cumplimiento de estos atributos aparentemente permite que la información proporcionada en los estados financieros sea útil para los usuarios. Estos atributos fueron incorporados en Colombia a través de un marco normativo robusto, y que está enmarcado en la emisión de la (Ley 1314, 2009) y de los decretos reglamentarios (ver figuras 1 y 2).
Las características cualitativas de la información financiera se agrupan de dos formas; la primera denominada "Cualidades de Mejora" conformada por los principios de Comparabilidad, Verificabilidad, oportunidad y Comprensibilidad, y en el segundo grupo, denominado "Cualidades fundamentales", conformada por los principios de Relevancia, Representación Fiel y Materialidad.

\section{- Cualidades de Mejora}

En relación con la comparabilidad, se establece que los usuarios deben poder comparar los estados financieros de una entidad a lo largo del tiempo para identificar

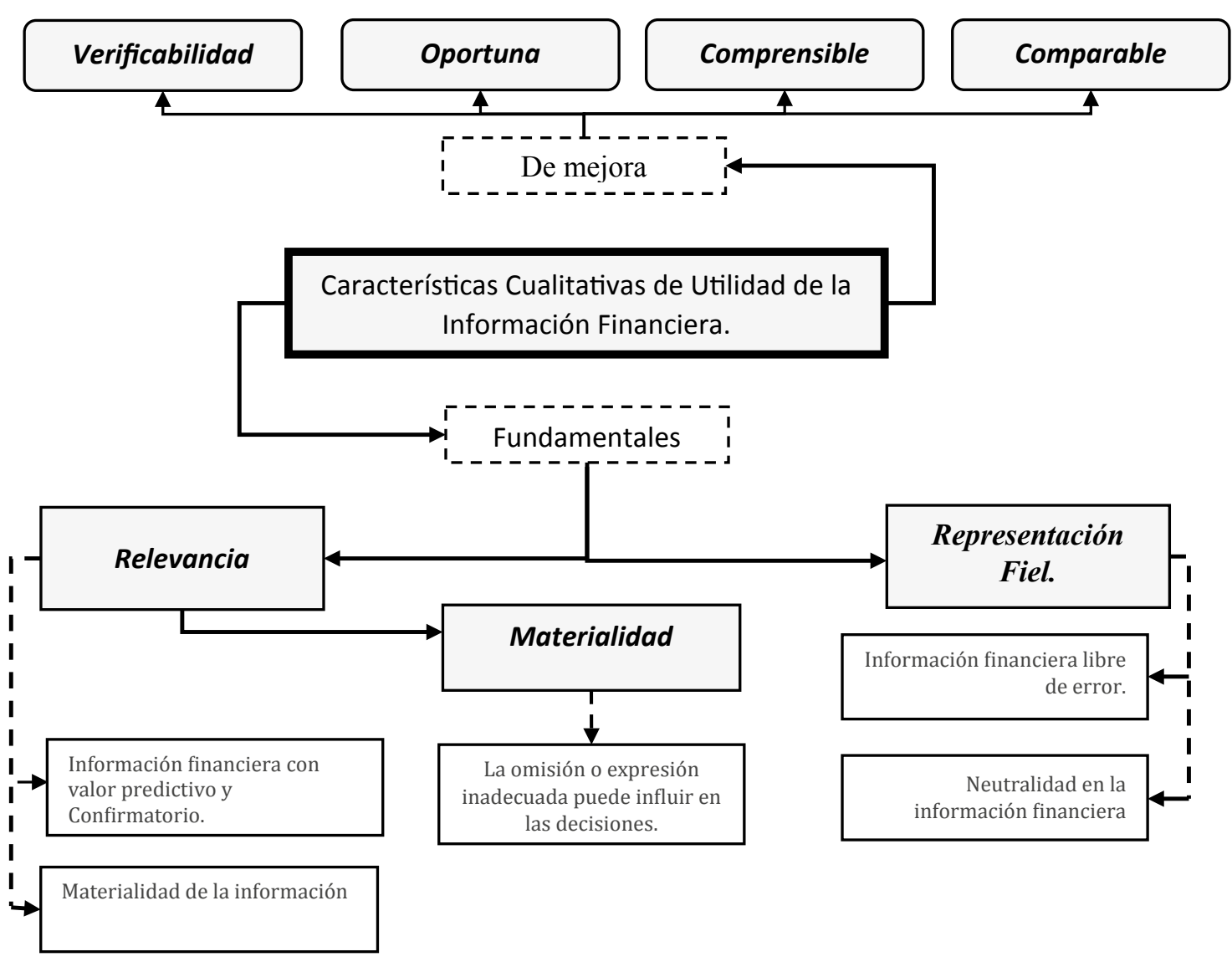

Figura 2 . Características de la Información Financiera en Colombia. Fuente: Elaboración propia a partir del Decreto 2420, 2015. 
tendencias en su posición financiera y rendimiento. Esto implica que los usuarios deben poder comparar los estados financieros de diferentes entidades para evaluar su posición financiera relativa, el rendimiento y los cambios en la posición financiera.

La Verificabilidad plantea que al reproducir y/o replicar los modelos, fórmulas y técnicas utilizados en el reconocimiento, medición, revelación y presentación de la información financiera, los usuarios de la información podrán lograr consensos, acuerdos y conclusiones similares a las expresadas en los hechos económicos. En ese sentido, se requiere de la disposición a tiempo de la información, para poder influir en sus decisiones (Oportunidad). El Decreto 2420 (2015) plantea que

... algunas explicaciones e información financiera con proyección al futuro no se pueden verificar hasta un periodo futuro, si es que se pueden verificar en algún momento. Para ayudar a los usuarios a decidir si quieren utilizar esa información" (p. 51).

Por su parte, Montilla, Montes y Mejía (2005) señalan que para darle cumplimiento al principio de comprensibilidad "los usuarios deben poseer un conocimiento razonable sobre los negocios, actividades económicas y contabilidad, sin embargo, no debe excluirse información por razones de dificultad para su comprensión"(pp. 67-68).

\section{- Cualidades Fundamentales}

El principio de confiabilidad plantea que la información debe estar libre de error material o sesgo y al cumplirse este principio los usuarios podrán utilizar la información como imagen fiel de la realidad que pretende representar o que se espera que represente. Has- ta este punto se podría establecer que para una organización resulta benéfico abordar la contabilidad financiera como un elemento de toma de decisión, sin embargo, con la implementación de las normas internacionales de información y en especial con las mediciones de activos y pasivos bajo el método de valor razonable se puede establecer que los resultados y en especial la información pueden verse distorsionada.

La información es relevante cuando es capaz de influir en las decisiones, es decir, tiene valor predictivo y/ confirmatorio. Sin embargo, la información es relevante en la medida que se exprese de manera adecuada y no se omitan partidas, transacciones o datos, es decir, se cumple con el principio de Materialidad. "En otras palabras la materialidad es un aspecto especifico de la Relevancia" (Decreto 2420, 2015, p. 49)

Para ser una representación fiel perfecta se deberá cumplir con tres características. La información debe ser completa en la media que incluya la descripción y explicaciones necesarias para los usuarios, neutral refleja de manera justa y sin sesgo los hechos económicos y libre de error.

\section{Usuarios de la Información Contable}

La alta calidad en la información financiera tiene la capacidad de satisfacer las necesidades explícitas e implícitas de los usuarios. A través de la información financiera los Inversionistas (Propietarios) buscan mejorar la capacidad de la organización para generar beneficios económicos, garantizar flujos de efectivo que permitan asumir las obligaciones de corto y largo plazo, para de esta manera garantizar la permanencia en el tiempo 
(Negocio en Marcha), las Entidades financieras buscan que sus operaciones de intermediación financiera (concesión de préstamos y créditos, negociación de efectivos, aseguramiento, inversión de capitales etc.) no se vean afectadas y se garanticen los retorno en los plazos y valores estimados y pactados.

Las entidades de control a través de la información financiera realizan inspección, control y vigilancia sobre temas de interés general, para de esta manera garantizar condiciones de estabilidad económica y social. Los Administradores de negocios busca que los procesos de producción, venta, calidad, suministro, financiamiento, entre otros, se desarrollen de manera apropiada.
Los Clientes pueden realizar análisis del manejo eficiente y eficaz de los recursos, permitiendo el acceso a créditos, mejores precios y calidad de los productos. Los Proveedores la garantía de los pagos oportunos y los Trabajadores en el aumento de retribuciones por su labor y la estabilidad laboral.

\section{Modelo empírico}

El cambio de las normas contables nacionales a las NIIF ha generado una "extensa literatura empírica sobre sus consecuencias económicas en los mercados de capital y la calidad de la información financiera" (Soderstrom \& Sun, 2007), por tal motivo, se propone un esquema
Usuarios de la Información Contable

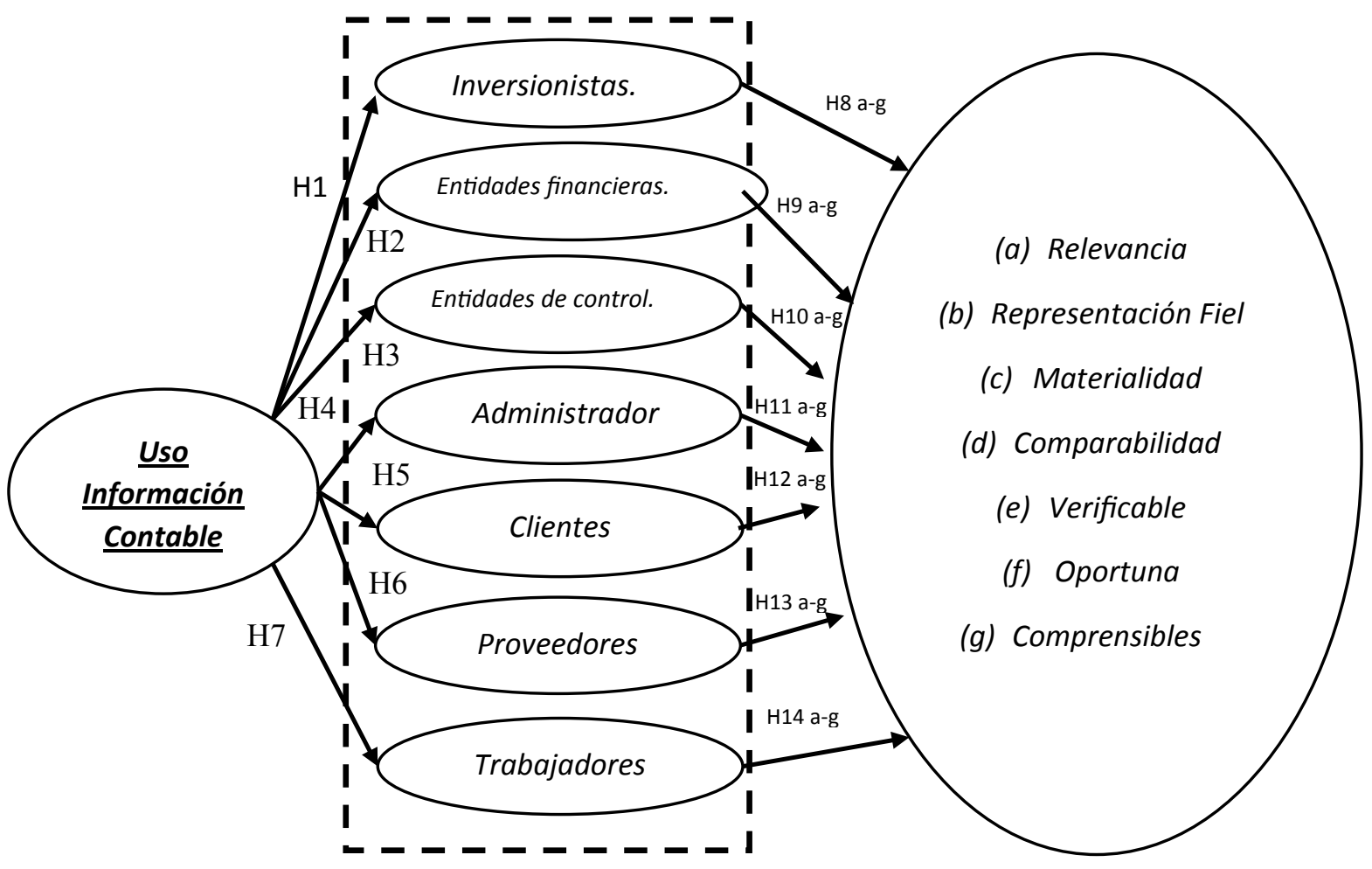

Figura 3. Esquema propuesto de análisis.

Fuente: Elaboración Propia a partir de marco normativo y teórico. 
que posibilite establecer como el uso de la información contable incide en la toma de decisiones de los usuarios.

Por su parte, la variación en la adopción de las NIIF presenta un problema para los investigadores al medir el nivel de adopción de las NIIF. La mayoría de las investigaciones revisadas sobre la adopción de las NIIF han medido el nivel de adopción teniendo en cuenta dos variables temporales: adopción "antes" y "después” de la implementación. La debilidad de estas mediciones radica en su incapacidad para medir con precisión el grado de adopción en cada país.
Sin embargo, para cumplir este objetivo se propone tener presente el marco normativo colombiano en relación a las características cualidades de la información contables en términos de mejora y cualidades fundamentales y segundo, brindar la posibilidad de verificar uno de 3 tipos de impactos según su forma; es decir, directo, indirecto y heterogéneo (ver figura 3)

A partir del esquema propuesto se pueden plantear 49 tipos de hipótesis o disertaciones. Para poder abordar dicho esquema se requiere establecer el posicionamiento filosófico, epistemológico y

TABLA 1

Herramientas Estadísticas.

\begin{tabular}{|c|c|c|}
\hline $\begin{array}{l}\text { Análisis } \\
\text { Estadístico }\end{array}$ & $\begin{array}{l}\text { Tipo de Variable } \\
\text { según Naturaleza }\end{array}$ & Herramientas \\
\hline \multirow{3}{*}{ Descriptiva } & Cualitativa & Porcentajes y Tasas (prevalencias, incidencias, etc.) \\
\hline & \multirow[b]{2}{*}{ Cuantitativas } & Medidas de tendencia central (Media, Mediana y Moda) \\
\hline & & $\begin{array}{l}\text { Medidas de dispersión (Varianza, desviación Estándar, } \\
\text { Rango y el Intervalo de confianza) }\end{array}$ \\
\hline \multirow{8}{*}{ Inferencial } & \multirow{2}{*}{ Cualitativa } & Test Chi Cuadrado de Pearson \\
\hline & & Prueba de probabilidad exacta de Fisher \\
\hline & \multirow{2}{*}{$\begin{array}{l}\text { Cuantitativa - } \\
\text { Paramétrica (Acorde a } \\
\text { la distribución normal) }\end{array}$} & T de Student \\
\hline & & Prueba ANOVA \\
\hline & $\begin{array}{l}\text { Cuantitativa No } \\
\text { paramétrica (No } \\
\text { manifiesta distribución } \\
\text { normal) }\end{array}$ & U de Mann - Whitney y Kruskal Wallis \\
\hline & \multirow{3}{*}{ Asociación } & Regresión (Múltiple o Logística) \\
\hline & & Correlación \\
\hline & & $\begin{array}{l}\text { Riesgo (La razón de momios - Odds Ration, Riesgo } \\
\text { Relativo - RR y el Numero Necesario a Tratar -NNT) }\end{array}$ \\
\hline
\end{tabular}

Fuente: Elaboración propia. 
ontológico, dado que esto incide en la forma del abordaje del problema y herramientas a utilizar. Desde la metodología cuantitativa es posible utilizar métodos estadísticos, estos se agrupan de dos formas: el análisis descriptivo y el inferencial. El análisis de tipo descriptivo busca en esencia Recolectar, Clasificar, ordenar, analizar y representar los resultados, por su parte la estadística Inferencial busca el análisis y la interpretación. Dependiente de la elección se tendrá que aplicar distintos tipos de herramientas (ver tabla 1 e ilustración 4). Desde la metodología cualitativa se pueden utilizar: la observación participante, grupo nominal, técnica Delphi, entrevistas, grupos de discusión e historia de vida.

\section{Conclusiones}

Teniendo en cuenta la transición reciente a las normas internacionales de información financiera y con el objeto de fortalecer y nutrir el desarrollo académico de la contabilidad, se propone un esquema que busca comprender como la adopción obligatoria de las Normas Internacionales de Información Financiera mejora la calidad de la información contable en Colombia. Para la elaboración de la propuesta se tuvo en cuenta: la evolución histórica e incorporación del concepto de calidad en términos del reconocimiento, medición, revelación y presentación de los estados financieros y las exigencias normativas colombianas en relación a las características cualitativas que debe cumplir la información contable.

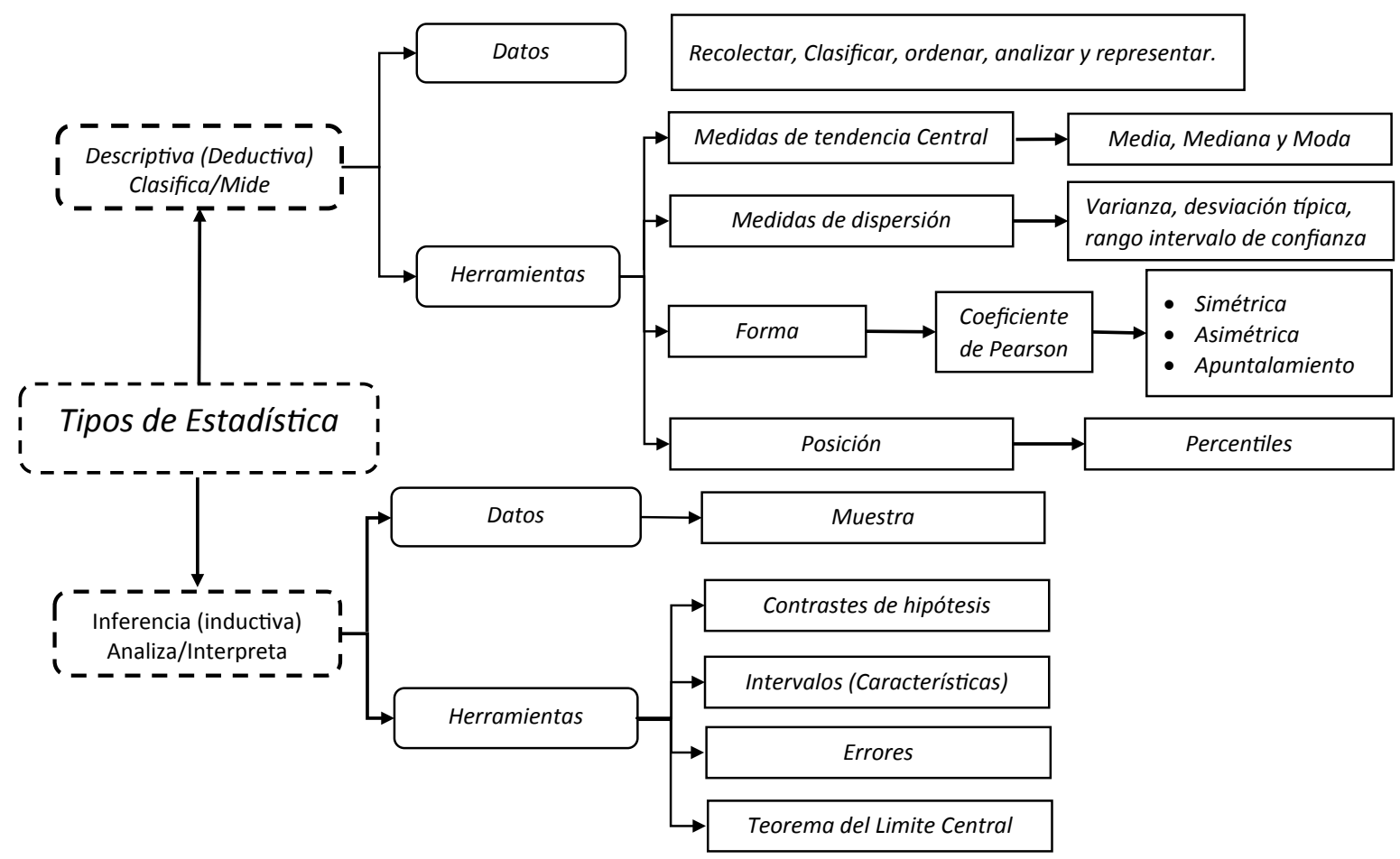

Figura 4. Tipos de estadística y alcance.

Fuente: Elaboración propia. 
Las investigaciones internacionales plantean una relación heterogénea entre la adopción de las normas internacionales y la calidad de la información contable, en razón a la complejidad y a los contextos legales, económicos, sociales y políticos de cada país, para el caso de Colombia las investigaciones en el tema son escasas y el marco normativo es extenso lo cual puede incidir en la interpretación y aplicación organizacional.

Y por último, dependiendo del posicionamiento filosófico, epistemológico y ontológico de la investigación se podrán utilizar herramientas de corte cuantitativo, basadas principalmente en las estadísticas y herramientas de corte cualitativa, que buscan interpretar y comprender contextos e interactuar con sociales complejos.

\section{REFERENCIAS}

Aguilar, P. y Medina, J. (2013). Administración y calidad de la información de los sistemas de información contable de las PYMES. Cuadernos de administracion, 29(49). 8-15.

Barbel, A. (2008). Las mediciones y la calidad de la informacion contable: un analisis desde la perspectiva de la regulacion contable internacional. Contabilidad y auditoria, 154-188.

Alvarado, L., Hernandez, J. y Chumaceiro, A. (2010). Calidad de servicio en universidades públicas venezolanas. TEACS, 2(4), 45-58.

Ball, R. (2001). Infrastructure requirements for an economically efficient system of public financial reporting and disclosure. Brookings-Wharton Papers on Financial Services, 4(1). 127-169.
Barth, M., Landsman, W. \& Lang, M. (2008). International Accounting Standards and Accounting Quality. Journal of Accounting Research, 446(3). 467-498. https://doi.org/10.1111/j.1475679X.2008.00287.x

Brown, P. (2011). International financial reporting standards: what are the benefits? Accounting and Business Research, 41(3). 269-285. https:// doi.org/10.1080/00014788.2011.569 054

Callao , S., Ferrer, C., Jarne, J. \& Laínez, J. (2010). IFRS adoption in Spain and the United Kingdom: Effects on Accounting numbers and relevance. Advances in Accounting, 26(2). 304-313. https:// doi.org/10.1016/j.adiac.2010.08.003

Cárdenas, A., Gallego, I. y Tobón, M. (2018). Percepción de la Calidad de la Información Contable del municipio de La Ceja. [Artículo de investigación]. Medellín: Universidad de Antioquia. Recuperado de https://www.academia. edu/29688432/Art\%C3\%ADculo_ de_investigaci \% C $3 \%$ B $3 \mathrm{n}$. Percepci\%C3\%B3n_de_la_Calidad_de_la_Informaci\% $\mathrm{C} 3 \% \mathrm{~B} 3 \mathrm{n}$ Contable_del_municipio_de_La_ Ceja

Chen, H., Tang, Q., Jiang, Y. \& Lin, Z. (2010). The role of International Financial Reporting Standards in Accounting Quality: evidence from the European Union. Journal of International Financial Management and Accounting, 21(3). 220-278. Recupeado de https://ssrn.com/abstract $=1330352$

Déniz, J., Bona, C., Pérez, A. y Suárez, H. (2007). Fundamentos de contabilidad financiera: teoría y práctica. Madrid: Delta. 
Gómez, V. y Ospina, Z. (2009). Ampliando las fronteras en la disciplina contable: una introducción para la contextualización de los ejemplares heterodoxos. En, M. Gómez-Villegas y C. Ospina-Zapata (eds., trads.). Avances insterdisciplinario para una comprensión crítica de la contabilidad (14-33). Medellin: Vieco e hijas.

González, C. (2009). Conceptos generales de calidad total. Buenos Aires: El Cid Editor.

Kvaal, E. \& Nobes, C. (2010). International differences in IFRS policy choice: a research note. Accounting and Business Research, 40(2). 173187. https://doi.org/10.1080/0001478 8.2010 .9663390

Montilla, O., Montes, C. y Mejía, E. (2005). Armonización o estandarización contable internacional. Cali: Universidad Libre.

Morais, A., Fialho, A. \& Dionísio, A. (2018). Is the accounting quality after the mandatory adoption of IFRS a random walk? Evidence from Europe. Journal of Applied Accounting Research, 19(3). 334-350. https:// doi.org/10.1108/JAAR-07-2016-0073

Morin, E. (2001). El método. La naturaleza de la naturaleza. Madrid: Catedra.

Nobes, C. (2006). The survival of international differences under IFRS: towards research agenda. Accounting and Business Research, 36(3). 233-245. https://doi.org/10.1080/000 14788.2006.9730023

Nobes, C. \& Stanbler, C. (2015). The quality characteristic of financial information , and managers accounting desicions: evidence from IFRS policy change. Accounting and Business Research, 45(5).572-601. https://doi. org/10.1080/00014788.2015.104449 5
Perafán, H. \& Benavides, J. (2017). Impact of IFRS on the quality of financial information in the United Kingdom and France: Evidence from a new perspective. Intangible Capital, 13(4). 850-878. http://dx.doi.org/10.3926/ ic. 939

Pérez, J. (1994). Gestión de la Calidad Empresarial. Madrid: Esic.

República de Colombia. Congreso de la República. (13 de julio de 2009). Por la cual se regulan los principios y normas de contabilidad e información financiera y de aseguramiento de información aceptados en Colombia, se señalan las autoridades competentes, el procedimiento para su expedición y se determinan las entidades. [Ley 1314]. DO: 47409. Recuperado de http://suin.gov.co/viewDocument. asp?ruta=Leyes $/ 1677255$

República de Colombia. Presidencia de la República. (14 de diciembre de 2015). Por medio del cual se expide el Decreto Único Reglamentario de las Normas de Contabilidad, de informacion financiera $y$ aseguramiento de la informacion y se dictan otras disposiciones. [Decreto 2420]. DO: 49726. Recuperado de http://www. suin-juriscol.gov.co/viewDocument. asp?ruta=Decretos $/ 30030273$

República de Colombia. Presidencia de la República. (30 de marzo de 1989). Por el cual se expide el Estatuto Tributario de los Impuestos Administrados por la Dirección General de Impuestos Nacionales. [Decreto 624]. DO: 38756. Recuperado de http://www.suin.gov. co/viewDocument.asp?id=1132325

Sanabria, P., Romero, V. y Flórez, C. (2013). El concepto de calidad en las organizaciones: una aproximación desde la complejidad. Universidad \& Empresa, 16(26). 157-205. https:// dx.doi.org/10.12804/rev.univ.empresa.27.2014.06 
Soderstrom, N. \& Sun, K. (2007). IFRS Adoption and Accounting Quality: A Review. European Accounting Review, 16(4). 36-37. https://doi. org/10.1080/09638180701706732

Villegas, M. (2003). Contabilidad: Comentarios sobre el discurso cientifico y los determinantes morales. Revista de ciencias administrativas y sociales, 22(2). 109-120.

Zorio, A., García, M. \& Pucheta, M. (2004). Calidad, un nuevo concepto clave en el desenlace del proceso de arminizacion contable internacional. Revista española de financiacion y Contabilidad, 33(121). 313-347.

\section{ANEXOS}

\section{Búsqueda estructurada}

La búsqueda de informacion esrtucturada se realizó mediante la utilización de bases de datos web que captan y distribuyen artículos de revistas científicas, libros y otras publicaciones.

\section{Biodata}

Julio Cesar Cantillo Padron es Magister en Ciencias Contables de la Universidad de Antioquia (Colombia) y Magister en Administración de la Universidad Nacional (Colombia). Especialista en gerencia de la Hacienda Pública y Contador Público de la Corporación Universitaria del Caribe (CECAR). https:// orcid.org/0000-0001-7589-7877

Lucelly Rivero González es Especialista en Proyectos de Desarrollo de la Escuela Superior de Administración Pública y Administradora de Empresas de la Universidad de Cartagena (Colombia). https://orcid.org/0000-0003-0156-0168 


\begin{tabular}{|c|c|}
\hline Herramienta: & Scopus \\
\hline Compañía: & Elsevier bv \\
\hline Función: & Buscador de información científica estructurada. \\
\hline Características & $\begin{array}{l}\text { Base de datos con más de: } \\
18.500 \text { revistas revisadas por colegas (incluyendo } 1.800 \text { revistas de acceso } \\
\text { abierto). } \\
400 \text { publicaciones comerciales. } \\
340 \text { libro de la serie. } \\
419 \text { actas de congresos. } \\
47 \text { millones de registros: } \\
26 \text { millones de registros con referencias anteriores a } 1996 \text { (de los cuales } 78 \% \text { son } \\
\text { referencias). } \\
21 \text { millones de registros anteriores a } 1996 \text { que se remontan hasta } 1823 \text {. } \\
4,9 \text { millones de documentos de la conferencia de los procedimientos y las revistas. }\end{array}$ \\
\hline Pagina Web & www.scopus.com \\
\hline Herramienta: & Science Direct \\
\hline Compañía: & ELSEVIER S.A. \\
\hline Función: & Buscador de información científica estructurada. \\
\hline Características & $\begin{array}{l}\text { Base de datos con más de: } \\
\text { Más de 2.500 Revistas. } \\
\text { Más de } 11.000 \text { Libros. } \\
\text { Más de } 11 \text { Millones de Artículos. }\end{array}$ \\
\hline Pagina Web & www.sciencedirect.com \\
\hline Herramienta: & Bussines Source Complete \\
\hline Compañía: & Ebsco industries.inc \\
\hline Función: & Buscador de información científica estructurada. \\
\hline Caracteristicas & $\begin{array}{l}\text { Base de datos con más de: } \\
175.000 \text { títulos de publicaciones periódicas. }\end{array}$ \\
\hline Página Web & www.Ebsco.com \\
\hline
\end{tabular}

\begin{tabular}{|l|l|}
\hline \multicolumn{1}{|c|}{ Herramienta: } & \multicolumn{1}{c|}{ Academic Search Complete } \\
\hline Compañía: & Elsevier bv \\
\hline Función: & Buscador de información científica estructurada. \\
\hline Características & $\begin{array}{l}\text { Base de datos con más de: } \\
\text { Base de datos académica de texto completo de diferentes áreas del conocimiento. } \\
\text { publicaciones arbitradas. Ofrece índices y resúmenes de más de 9.990 } \\
\text { publicaciones especializadas y un total de más 10.400 publicaciones diversas, } \\
\text { entre las que se incluyen monografías, informes y conferencias. }\end{array}$ \\
\hline Plataforma & EBSCO \\
\hline
\end{tabular}




\begin{tabular}{|c|c|c|c|c|}
\hline Bases de Datos de Búsqueda & Tipo & Resulta & enidos & Ecuación de Búsqueda \\
\hline \multirow{5}{*}{ Scopus } & \multirow{5}{*}{$\begin{array}{l}\text { Herramienta } \\
\text { bibliográfica }\end{array}$} & 2017 & 1 & \multirow{19}{*}{$\begin{array}{l}\text { "Quality of financial } \\
\text { statement information" }\end{array}$} \\
\hline & & 2014 & 2 & \\
\hline & & 2012 & 1 & \\
\hline & & 2010 & 1 & \\
\hline & & 2008 & 1 & \\
\hline \multirow{5}{*}{ Science Direct } & \multirow{5}{*}{$\begin{array}{l}\text { Base de } \\
\text { datos }\end{array}$} & 2016 & 2 & \\
\hline & & 2015 & 5 & \\
\hline & & 2014 & 1 & \\
\hline & & 2011 & 31 & \\
\hline & & 2010 & 3 & \\
\hline \multirow{4}{*}{ Business Source Complete } & \multirow{4}{*}{$\begin{array}{l}\text { Base de } \\
\text { datos }\end{array}$} & 2014 & 1 & \\
\hline & & 2012 & 1 & \\
\hline & & 2010 & 1 & \\
\hline & & 2008 & 1 & \\
\hline \multirow{5}{*}{ Academic Search Complete } & \multirow{5}{*}{$\begin{array}{l}\text { Base de } \\
\text { datos }\end{array}$} & 2018 & 1 & \\
\hline & & 2017 & 4 & \\
\hline & & 2016 & 7 & \\
\hline & & 2015 & 4 & \\
\hline & & 2014 & 2 & \\
\hline
\end{tabular}

